\title{
PREDIÇÃO DA PRODUÇÃO DE MULTIPRODUTOS EM NÍVEL DE ÁRVORE INDIVIDUAL DE Pinus taeda L.
}

\author{
PREDICTING MULTIPRODUCT PRODUCTION AT THE INDIVIDUAL TREE LEVEL OF \\ Pinus taeda $L$.
}

\author{
Ricardo Cavalheiro ${ }^{1}$, Sebastião do Amaral Machado ${ }^{1}$, Afonso Figueiredo Filho', \\ Allan Libanio Pelissari ${ }^{1}$ \\ ${ }^{1}$ Universidade Federal do Paraná, Curitiba, Paraná, Brasil - cavalheiro1@hotmail.com, \\ samachado@ufpr.br\&allanpelissari@gmail.com \\ ${ }^{2}$ Universidade Estadual do Centro Oeste, Irati, Paraná, Brasil -afigfilho@gmail.com
}

\begin{abstract}
RESUMO
Modelos de árvores individuais permitem o maior nível de detalhamento possível da estrutura florestal e estudos recentes mostram que ainda existem lacunas de conhecimento principalmente quando se planeja obter a segmentação em multiprodutos. O objetivo deste trabalho foi ajustar um modelo completo de árvores individuais para estimar os volumes por sortimentos em plantios de Pinus taeda localizados no planalto norte do estado de Santa Catarina. O ajuste do sistema foi baseado nas remedições de 345 parcelas permanentes. Para estimativa dos sortimentos utilizou-se o modelo de Schoepfer (1966) e a cubagem rigorosa de 360 árvores. A mortalidade por hectare foi estimada a partir do ajuste do modelo de Buchman. O índice de competição independente da distância utilizado foi o de Stage (1973). O modelo proposto por Qin \& Cao (2006) foi ajustado para projeção do diâmetro à altura do peito e altura total. Para a avaliação dos modelos, foram utilizadas o coeficiente de determinação ajustado ( $\mathrm{R}^{2} \mathrm{adj}$ ), erro padrão da estimativa $\left(S_{y x \%}\right)$ e análise gráfica de resíduos. Utilizou-se o teste de Mann-Whitney para avaliar se os volumes estimados apresentam igualdade estatística quando comparados com os volumes observados. Os resultados do ajuste probabilidade de mortalidade foram $u m R^{2}$ adj $=0,623$ e $S_{Y x \%}=41,9$. Os modelos de estimativas de diâmetro e altura total apresentaram respectivamente $u m R^{2}{ }_{\text {adj }}=0,987, R^{2}$ adj $=0,963$ e $S_{Y X \%}=2,22, S_{Y X \%}=4,04$. 0 volume foi superestimado para todos os sortimentos em média $43,6 \mathrm{~m}^{3}$.ha-1 $(29,8 \%)$. Quanto a acurácia os volumes diferiram em média em $45,6 \mathrm{~m}^{3}$.ha-1 $(31,3 \%)$ dos volumes reais. O resultado do teste de Mann- Whitney mostrou que existiu diferença estatística entre os valores observados e estimados. O sistema de árvores individuais construído não apresenta projeções precisas e acuradas. Também gera-se estimativas que diferem estatísticamente dos volumes observados para todos os sortimentos.
\end{abstract}

PALAVRAS-CHAVE: Índice de competição, Modelagem, Modelo completo, Mortalidade, Sortimento.

\section{ABSTRACT}

Individual tree models allow the greatest possible level of detail of the forest structure. Recent studies show that knowledge gaps still exist, especially when planning the obtaintion of multiproducts. The objective of this work was to adjust a complete model of individual trees to estimate volumes by assortments in Pinus taeda plantations located in the northern plateau of the State of Santa Catarina. The adjustment of the system was based on the remeasurement of 345 permanent plots. The Schoepfer model (1966) and the rigorous cubage of 360 trees were used to estimate the assortments. Mortality per hectare was estimated by adjusting the Buchman model. The competition index independent of the distance used was that from Stage (1973). The model proposed by Qin \& Cao (2006) was adjusted to project the diameter at breast height and total height. The adjusted determination coefficient $\left(R^{2}{ }_{a d j}\right)$, standard error of the estimate $\left(\mathrm{S}_{\mathrm{YX} \%}\right)$ and graphical analysis of residuals were used to evaluate the models. The MannWhitney test was used to assess whether the estimated volumes are statistically equal when compared to the observed volumes. The results of the mortality model were $R^{2}{ }_{\text {adj }}=0.623$ and $\mathrm{S}_{\mathrm{YX} \%}=41.9$. The models of diameter and total height estimates showed respectively $R^{2}$ adj $=0.987, R^{2}{ }_{\text {adj }}=0.963$ and $S_{Y X \%}=2.22, S_{Y X \%}=4.04$. The volume was overestimated for all assortments being overestimated by an average of $43.6 \mathrm{~m}^{3}$.ha-1 $(29.8 \%)$. As for accuracy, volumes differed on average $45.6 \mathrm{~m}^{3}$.ha-1 $(31.3 \%)$ from the observed volumes. The result of the Mann-Whitney test showed that there was a statistical difference between the observed and estimated values. The system of individual trees built did not present precise and accurate projections. The generated estimates also differ statistically from the observed volumes for all assortments. 


\section{INTRODUÇÃO}

Empresas de base florestal monitoram o incremento de seus ativos de modo a planejar as atividades do empreendimento no curto, médio e longo prazo. Entender a dinâmica de crescimento das florestas é impescindível para guiar as estratégias da empresa desde o dimensionamento da mão-de-obra até a oferta do produto madeireiro para clientes internos e ou externos.

Para obter estimativas confiáveis de estoques de madeira presentes e futuros, faz-se o uso de modelos de crescimento e produção. Esses modelos podem ser constituídos de uma ou mais equações, sendo essenciais na avaliação de alternativas de manejo. Em função do nível de resolução, os modelos de crescimento e produção podem ser classificados em nível de povoamento, distribuição por classes de diâmetro e em nível de árvores individuais (CAMPOS \& LEITE, 2017).

Ao considerar empresas focadas na oferta de um único produto da floresta, modelos ao nível de povoamento são capazes de fornecer as informações necessárias para a tomada de decisão do gestor florestal (BURKHART \& TOMÉ, 2012). Quando se tem por objetivo a oferta de multiprodutos, existe a necessidade da adoção do uso de modelos que possam disponibilizar um grau de detalhamento maior do ativo florestal.

Modelos de árvores individuais permitem o maior nível de detalhamento possível da estrutura florestal, uma vez que a produção é estimada por árvore ao longo do tempo (CAO, 2017). Essa categoria de modelos apresenta três componentes básicos: um modelo de estimativa da mortalidade, um modelo de crescimento em diâmetro e um modelo de crescimento em altura total (BURKHART \& TOMÉ, 2012).

De acordo com Pretzsch (2009), os primeiros registros de estudos da modelagem de árvores individuais ocorreram a partir do ano de 1964. No entanto, estudos recentes mostram que ainda existem lacunas de conhecimento para esse tema. Miranda (2016) desenvolveu o primeiro modelo completo de crescimento e produção ao nível de árvores individuais para Pinus taeda no Brasil. Segundo esse autor, as estimativas do volume total por hectare foram estatisticamente iguais aos valores observados nos povoamentos localizados na região de Telêmaco Borba, estado do Paraná.

Téo (2017) ajustou um sistema fundamentado no crescimento individual das árvores, para plantios desbastados e não desbastados de Pinus taeda, na região Meio Oeste do estado de Santa Catarina. Os modelos ajustados ppor esse autor para estimar probabilidade da mortalidade, incremento em diâmetro e incremento em altura total apresentaram-se adequados para composição de todo o sistema de modelagem ao nível de árvore individual.

Scolforo et al. (2019) desenvolveram um modelo compatível de árvores individuais e modelo global para plantios clonais de Eucalipto distribuídos em quatorze sítios distintos do Brasil. De acordo com os resultados apresentados, os modelos de árvores individuais geraram estimativas acuradas. O sistema desenvolvido forneceu um nível detalhado de informação permitindo a extração de informações do volume ao nível de multiprodutos.

Tendo em vista o grau de detalhamento que a modelagem de árvores individuias oferece, bem como a necessidade de algumas empresas na segmentação em multiprodutos dos volumes provenientes de plantios de Pinus taeda, torna-se imprescindível estudos que desenvolvam modelos capazes de estimar de forma precisa e acurada a produção final ao nível de sortimentos.

Com isso, o objetivo deste trabalho foi ajustar um modelo completo de árvores individuais para estimar os volumes por sortimentos em plantios de Pinus taeda localizados no planalto norte do estado de Santa Catarina.

\section{MATERIAL E MÉTODOS}

\section{Origem dos dados}

Os dados para o desenvolvimento desta pesquisa foram disponibilizados por uma empresa florestal localizada no Planalto Norte do estado de Santa Catarina conforme Figura 1.

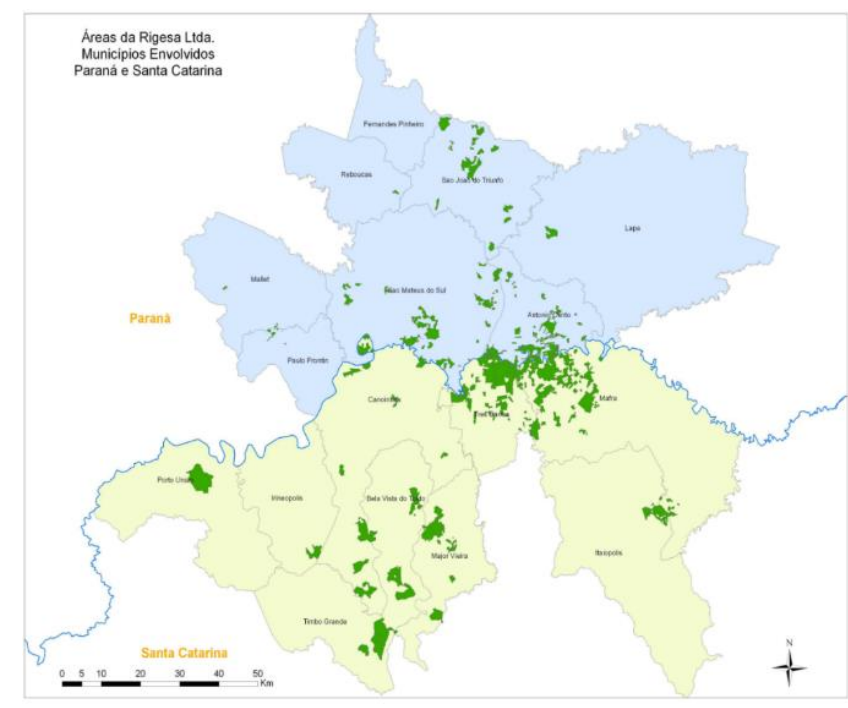

Figura 1. Distribuição das áreas dos povoamentos de Pinus taeda. 
Nessa região o clima é do tipo Mesotérmico Úmido (Cfb) segundo a classificação de Köppen, em que a temperatura média do mês mais quente é inferior a $22^{\circ} \mathrm{Ce}$ a do mês mais frio inferior a $18^{\circ} \mathrm{C}$. A precipitação tem distribuição equilibrada em todos os meses, com média de $1.800 \mathrm{~mm}$ por ano e sem déficit hídrico.

$\mathrm{O}$ ajuste do sistema foi baseado nas remedições anuais ou bianuais de 345 parcelas permanentes alocadas em povoamentos de Pinus taeda sem desbaste, com dimensões de $20 \mathrm{~m} \times 20 \mathrm{~m}$. As medições ocorreram ao longo de 24 anos, sendo que a amplitude de idade foi de 5 a 22 anos. Ao final, considerando todas as remedições, o total de parcelas disponíveis para a realização deste trabalho foi de 2.641, totalizando 152.378 árvores com os diâmetros à altura do peito e as alturas totais mensuradas.

Dentre as 345 parcelas disponíveis, foram sorteadas 89 parcelas para compor a base de dados de validação, as quais não participaram da etapa dos ajustes dos modelos. A primeira medição dessas parcelas de validação foi utilizada para a estimativa anual da probabilidade de mortalidade por classe de diâmetro, incremento em diâmetro e incremento em altura total. Quando a projeção das variáveis da parcela alcançou a última medição próxima da idade de rotação, calculou-se os volumes por sortimento ao nível de árvore individual. A agregação desses volumes estimados resultou no volume total por hectare, o qual foi comparado com os valores observados.

Os sortimentos utilizados foram denomidados de "Celulose" com diâmetros entre 08 e 17,99 cm, "Tora I" com diâmetros entre 18 e 24,99 cm e "Tora II" com diâmetros maiores que $25 \mathrm{~cm}$. O comprimento de cada seção considerado foi de $2,65 \mathrm{~m}$.

\section{Índice de sítio}

A altura dominante de cada parcela foi obtida pelo critério proposto por Assmann (1970) a partir da altura média das 100 árvores de maior diâmetro por hectare. 0 modelo (1) utilizado para estimativa da altura dominante em função da idade foi o de Chapman-Richards (RICHARDS, 1959; CHAPMAN, 1961):

$$
h_{d o m}=\beta_{0}\left[1-e^{\left(-\beta_{1} I\right)}\right]^{\left[\left(1-\beta_{2}\right)^{-1}\right]}
$$

Em que: $h_{\text {dom }}=$ altura dominante $(\mathrm{m}) ; I=$ idade do povoamento (anos).

Para o ajuste da equação, foram utilizados 2.641 pares de dados com idade variando entre 5 e 22 anos. A idade de referência adotada foi de 17 anos conforme idade técnica de rotação adotada pela empresa.

\section{Equação de afilamento}

Para estimativa dos sortimentos utilizou-se o modelo de Schoepfer (1966) e a cubagem rigorosa de 360 árvores selecionadas com base nas idades e na distribuição diamétrica das parcelas. O coeficiente de determinação ajustado para essa equação foi de 0,987 e o erro padrão da estimava foi de $2,1 \%$.

$$
\begin{array}{rl}
\frac{d_{i}}{D A P}=1,1963-3,6 & 613\left(\frac{h_{i}}{H}\right)+14,6316\left(\frac{h_{i}}{h t}\right)^{2} \\
& -31,1455\left(\frac{h_{i}}{h t}\right)^{3}+29,5105\left(\frac{h_{i}}{h t}\right)^{4} \\
& -10,5414\left(\frac{h_{i}}{h t}\right)^{5}
\end{array}
$$

Em que: $d_{i}=$ diâmetro ao longo do fuste $(\mathrm{cm})$; $D A P=$ diâmetro a $1,30(\mathrm{~cm}) ; h_{i}=$ altura ao longo do fuste $(\mathrm{m}) ; h t=$ altura total da árvore $(\mathrm{m})$.

Para todas as estapas de cálculo do volume individual por sortimento de madeira, integrou-se a equação de afilamento ajustada.

\section{Probabilidade de mortalidade}

A probabilidade de mortalidade atual das parcelas foi obtida pela expressão (3):

$$
P m a=N_{m}\left(N_{v}+N_{m}\right)^{-1}
$$

Em que: Pma = probabilidade de mortalidade atual por classe diamétrica; $N_{m}=$ número de árvores mortas da parcela por hectare; $N_{v}=$ número de árvores vivas da parcela por hectare.

O índice de competição independente da distância utilizado foi o de Stage (1973) representado pela seguinte expressão (4):

$$
I C=\left(d_{i} q^{-1}\right)^{2}
$$

Em que: $I C$ = Índice de competição; $d_{i}=\mathrm{DAP}$ da árvore objeto $(\mathrm{cm}) ; q=$ diâmetro quadrático das árvores da unidade amostral $(\mathrm{cm})$.

A mortalidade por hectare foi estimada a partir do ajuste do modelo de estimativa da probabilidade de 
mortalidade por classe diamétrica proposto por Buchman (5):

$$
P m=\beta_{0}+\left(1+e^{\beta_{0}+\beta_{1} I C_{i}+\beta_{2} C C_{d}+\beta_{3} h_{m}+\beta_{4} I+\beta_{5} G_{c}+\beta_{6} S}\right)^{-1}
$$

Em que: $P m$ = probabilidade de mortalidade por classe diamétrica; $I C_{i}=$ índice de competição; $C C_{d}=$ centro da classe diamétrica $(\mathrm{cm}) ; h_{m}=$ altura total média da classe diamétrica $(\mathrm{m}) ; I=$ idade da parcela (anos); $G_{c}=$ área basal da classe diamétrica $\left(\mathrm{m}^{2}\right.$.ha ${ }^{-1}$.classe); $S=$ índice de sítio (m); $\beta_{i}=$ coeficientes a serem estimados.

\section{Modelo de projeção do diâmetro à altura do peito e altura total}

O modelo proposto por Qin \& Cao (2006) foi aplicado para projeção do diâmetro à altura do peito e altura total conforme formulação (6):

$$
Y_{2}=Y_{1}+e^{\left(\beta_{0}+\frac{\beta_{1}}{I_{2}}+\beta_{2} R S_{1}+\beta_{3} G_{1}+\beta_{4} Y_{1}\right)}
$$

Em que: $Y_{2}$ e $Y_{1}=$ variável de interesse (diâmetro e altura total) na idade atual e de projeção, respectivamente; $I_{2}=$ idade de projeção (anos); $R s=$ espaçamento relativo, $R s=\left[(10.000 / N)^{0,5} /\right.$ hdom $]$ sendo a proporção da distância média entre duas árvores assumindo espaço quadrático; $G_{1}=$ área basal ( $\mathrm{m}^{2}$.ha-1 $)$ na idade atual; e $\beta_{i}=$ coeficientes a serem estimados.

\section{Avaliação de modelos de regressão ajustados}

Para a avaliação dos modelos ajustados pelo método de regressão, foram utilizadas as seguintes estatísticas: coeficiente de determinação ajustado ( $R^{2}$ adj), erro padrão da estimativa $\left(S_{y x \%}\right)$ e análise gráfica de resíduos.

\section{Avaliação dos volumes projetados}

A distribuição dos volumes projetados por sortimento seguiu uma distribuição não normal, por isso, optou-se por utilizar o teste de Mann-Whitney com objetivo de avaliar se os volumes estimados pelo sistema de modelagem de árvores individuais apresentam igualdade estatística quando comparados com os volumes observados. Testouse a seguinte hipótese ao nível de $5 \%$ de significância:

$$
\begin{aligned}
& H_{0}: V o l_{\text {obs }}=\operatorname{Vol}_{\text {est }} \\
& H_{1}: \operatorname{Vol}_{\text {obs }} \neq \operatorname{Vol}_{\text {est }}
\end{aligned}
$$

Em que: $H_{0}=$ mediana dos volumes observados é igual a mediana dos volumes estimados; $\mathrm{Vol}_{\text {obs }}=$ volumes observados; $\mathrm{Vol}_{\text {est }}=$ volumes estimados; $H_{1}=$ hipótese alternativa.

Para verificar tendenciosidade das estimativas dos volumes projetados por sortimento, aplicou-se a análise de Viés e Acurácia. Esses critérios foram calculados pela seguinte formulação:

$$
\begin{gathered}
\text { Viés }=\frac{1}{n} \sum_{i=1}^{n}\left(y_{i}-\widehat{y}_{l}\right) \\
\text { Acurácia }=\frac{1}{n} \sum_{i=1}^{n}\left|y_{i}-\widehat{y}_{l}\right|
\end{gathered}
$$

Em que: Viés = também conhecido por tendenciosidade ou "bias"; Acurácia = mede em termos absolutos a proximidade de cada observação ao valor-alvo que se procura atingir; $y_{i}=$ valor observado; $\widehat{y}_{l}=$ valor estimado pela equação; $\bar{y}=$ valor médio da variável observada; $n$ = número de observações.

\section{RESULTADOS E DISCUSSÃO}

O modelo ajustado para estimativa da altura dominante apresentou resultados satisfatórios conforme exposto na Tabela 1. Observa-se que os coeficientes e as estatísticas de regressão dos ajustes apresentaram-se adequadas com $\mathrm{R}^{2}{ }_{\text {adj }}=0,8767$ e $\mathrm{S}_{\mathrm{y} \%}=8,20$, resultados consistentes $\mathrm{e}$ similares aos apresentados por MIRANDA (2016) com SYX\% $=9,50$.

Tabela 1. Coeficientes e estatística do modelo utilizado para estimar a altura dominante em povoamento de Pinus taeda.

\begin{tabular}{ccc}
\hline Coeficientes & $\mathbf{R}^{2}$ adj & SYX\% \\
\hline$b_{0}=42,2482^{*}$ & & \\
$b_{1}=0,0593^{*}$ & 0,8767 & 8,20 \\
$b_{2}=0,1001^{*}$ & & \\
\hline
\end{tabular}

$\mathrm{R}^{2}$ adj $=$ coeficiente de determinação ajustado; $\mathrm{S}_{\mathrm{xy} \%}=$ erro padrão da estimativa (\%); $\beta_{\text {is }}=$ coeficientes estimados; * coeficientes significativos a $5 \%$.

Conforme a Figura 2, as alturas dominantes foram superestimadas nas idades 5 e 6 anos pela equação ajustada. Nas outras idades, o modelo apresentou distribuição de resíduos com uma leve tendência de superestimativa com erros variando entre $+20 \%$ e $-40 \%$. 


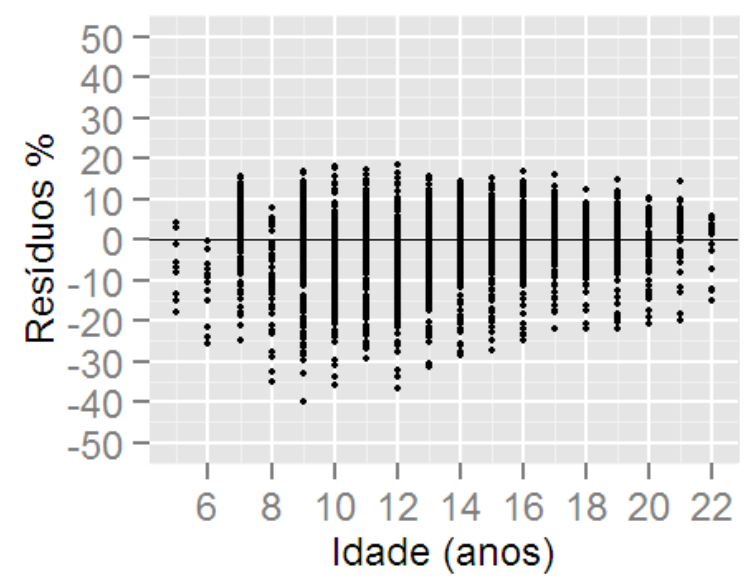

Figura 2. Distribuição de resíduos para relação altura dominante $\mathrm{x}$ idade em povoamentos de Pinus taeda.

As curvas de sítio foram construídas com o modelo ajustado. A amplitude utilizada foi de $3,5 \mathrm{~m}$ na idade de referência de 17 anos, cobrindo assim todas as parcelas em 4 classes de sítios conforme a Figura 3.

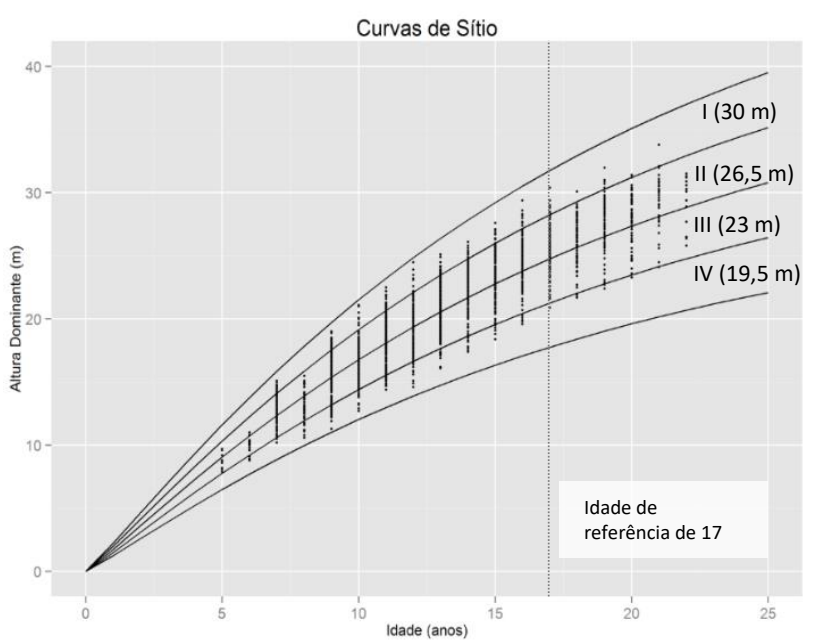

Figura 3. Curvas de sítio construídas com a equação de Chapman e Richards para povoamentos de Pinus taeda.

As estatísticas dos modelos ajustados para a construção do sistema de crescimento são apresentadas na Tabela 2. A modelagem estimativa da probabilidade de mortalidade apresentou alta complexidade já que a sobrevivência e a mortalidade de uma árvore estão associadas há vários fatores e indíviduos com as mesmas características podem apresentar outputs diferentes (FIORENTIN et al., 2019). Para essa variável os resultados do ajuste foram satisfatórios com $\mathrm{R}^{2}$ adj $=0,623$ e $\mathrm{S}_{\mathrm{y}} \%=41,9$. Resultados superiores quando comparados aos apresentados por Miranda et al. (2017) que com o uso do mesmo modelo em povoamentos de Pinus taeda, obteve um Syx\% $_{y}=393,1$. As estatísticas para os modelos de projeção em diâmetro e altura total apresentaram-se adequadas com respectivos $R^{2}{ }_{\text {adj }}=0,987, R^{2}{ }_{\text {adj }}=0,963$ e $S_{Y x}=2,22, S_{Y x}=4,04$.

Tabela 2. Coeficientes e estatísticas dos modelos testados para estimativa da probabilidade de mortalidade, projeção do diâmetro e da altura total em povoamentos de Pinus taeda.

\begin{tabular}{|c|c|c|c|c|}
\hline Autor/Modelo & & eficientes & R2 aj & $\mathrm{S}_{\mathrm{YX}} \%$ \\
\hline \multirow{8}{*}{$\begin{array}{l}\text { Probabilidade de Mortalidade } \\
\text { (Buchman) }\end{array}$} & b0 & $0,2289 *$ & \multirow{8}{*}{0,623} & \multirow{8}{*}{41,98} \\
\hline & b1 & $2,4768^{*}$ & & \\
\hline & b2 & $-0,4674^{*}$ & & \\
\hline & b3 & $-0,1764 *$ & & \\
\hline & b4 & $0,0493^{*}$ & & \\
\hline & b5 & $-0,1159 *$ & & \\
\hline & b6 & $3,7338^{*}$ & & \\
\hline & b7 & $-0,0650 *$ & & \\
\hline \multirow{5}{*}{$\begin{array}{l}\text { Predição do Diâmetro (CAO, } \\
\text { 2006) }\end{array}$} & b0 & $-2,5090^{*}$ & \multirow{5}{*}{0,987} & \multirow{5}{*}{2,22} \\
\hline & b1 & $18,9026^{*}$ & & \\
\hline & b2 & $-1,0818^{*}$ & & \\
\hline & b3 & $-0,0205^{*}$ & & \\
\hline & b4 & $0,0841^{*}$ & & \\
\hline \multirow{5}{*}{$\begin{array}{l}\text { Predição da Altura Total (CAO, } \\
\text { 2006) }\end{array}$} & b0 & $-1,1329 *$ & \multirow{5}{*}{0,963} & \multirow{5}{*}{4,04} \\
\hline & b1 & $17,2365^{*}$ & & \\
\hline & b2 & $-3,3936 *$ & & \\
\hline & b3 & $0,0052 *$ & & \\
\hline & b4 & $0,0139 *$ & & \\
\hline
\end{tabular}

* coeficientes significativos a $5 \%$.

Na Figura 4 está a distribuição dos resíduos das estimativas obtidas pelos ajustes dos modelos que fizeram parte do sistema de modelagem de árvores individuais. No geral, a probabilidade de mortalidade apresentou tendenciosidade de subestimativas sendo que em algumas idades os erros foram maiores que $200 \%$. Observa-se que as estimativas de diâmetro ficaram bem distribuídas com resíduos entre +/- $25 \%$. Por outro lado, a estimativa da altura total apresentou tendenciosidade de superestimativa principalmente nas idades inferiores aos 15 anos.

As estatísticas da projeção do atributo volume são apresentadas na Tabela 3. Para o sortimento de Celulose o volume foi superestimado em média $28,9 \mathrm{~m}^{3}$.ha-1 (10,3\%). Já para Tora I foi superestimado em média $54,3 \mathrm{~m}^{3} \cdot \mathrm{ha}^{-1}$ $(17,8 \%)$. As estimativas Tora II foram as que apresentaram o maior viés médio com superestimatimas em média de $47,6 \mathrm{~m}^{3} \cdot \mathrm{ha}^{-1}(61,3 \%)$. 

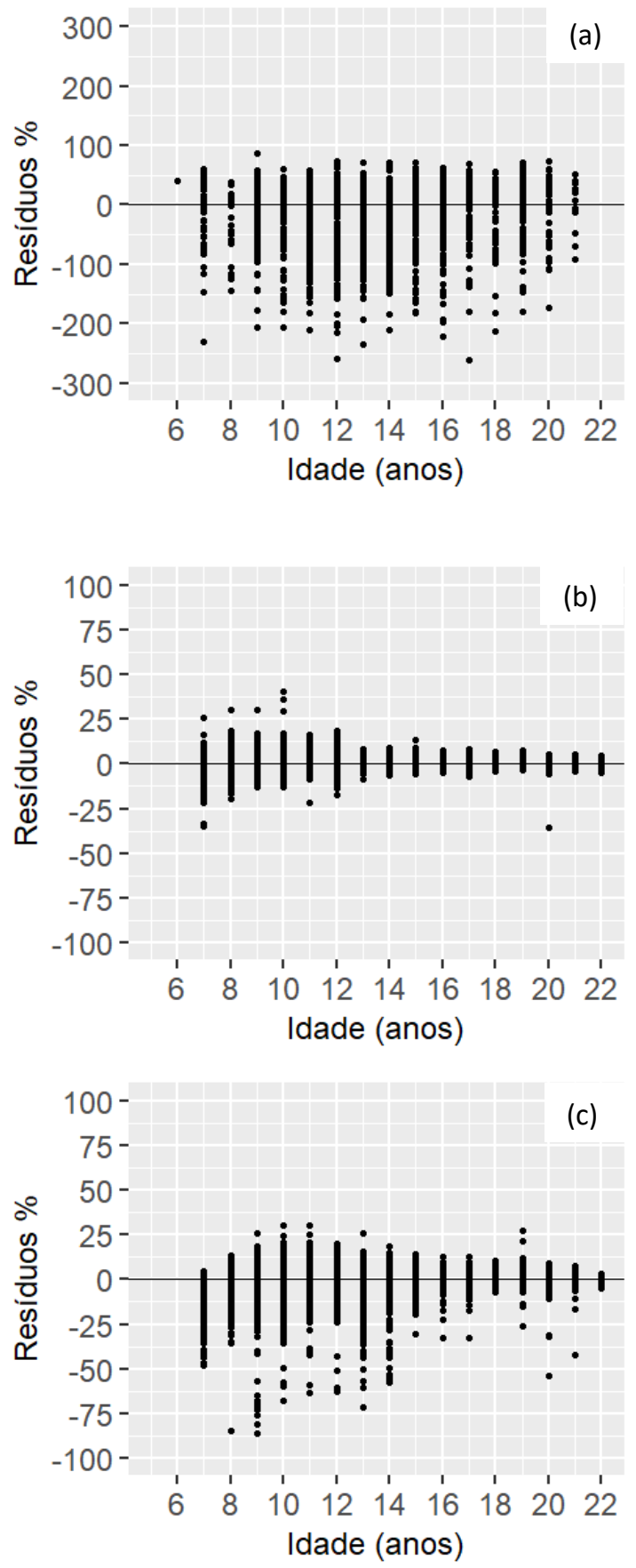

Figura 4. Distribuição de resíduos dos modelos ajustados para estimar a probabilidade de mortalidade (a), projeção do diâmetro (b) e da altura total (c) nos povoamentos de Pinus taeda.

Quanto a acurácia, os melhores resultados também foram para estimativa da celulose, onde os volumes diferiram em média em $30,6 \mathrm{~m}^{3}$.ha-1 $(11,1 \%)$ dos volumes reais. As estimativas menos acuradas foram geradas para 0 sortimento Tora II, com uma acurácia média de $50,4 \mathrm{~m}^{3} \cdot \mathrm{ha}^{-1}$, equivalendo a $64,5 \%$.
Tabela 3. Estatísticas da projeção do volume por sortimento nos povoamentos de Pinus taeda.

\begin{tabular}{cccc}
\hline Estatística & Celulose & Tora I & Tora II \\
\hline Viés $\left(\mathrm{m}^{3} \cdot \mathrm{ha}^{-1}\right)$ & $-28,9$ & $-54,3$ & $-47,6$ \\
Viés \% & $-10,3$ & $-17,8$ & $-61,3$ \\
Acurácia $\left(\mathrm{m}^{3} \cdot \mathrm{ha}^{-1}\right)$ & 30,6 & 55,9 & 50,4 \\
Acurácia \% & 11,1 & 18,5 & 64,5 \\
\hline
\end{tabular}

Na Tabela 4, apresenta-se os resultados do teste de Mann- Whitney da estimativa de volume para diferentes sortimentos. A hípotese nula foi rejeitada para todos os multiprodutos, ou seja, existiu diferença estatística entre os valores observados e estimados ao nível de $5 \%$ de significância.

Tabela 4. Resultados do teste de Mann Whitney para estimativa de sortimentos em plantios de Pinus taeda.

\begin{tabular}{|c|c|c|c|c|c|}
\hline \multirow{2}{*}{ Sortimento } & \multirow{2}{*}{$\mathbf{N}$} & \multicolumn{2}{|c|}{ Mediana (ton.ha-1) } & \multirow{2}{*}{ w } & \multirow{2}{*}{ p-valo } \\
\hline & & Observado & Estimado & & \\
\hline Celulose & 89 & 266,7 & 285 & 6648 & 0,000 \\
\hline Tora I & 89 & 305,8 & 377,8 & 6088 & 0,000 \\
\hline Tora II & 89 & 85,5 & 139,7 & 6400 & 0,000 \\
\hline
\end{tabular}

Na Figura 5 observa-se os volumes estimados em função dos volumes observados considerando todos os sortimentos. Os volumes de Celulose foram tendenciosos em valores acima de $300 \mathrm{~m}^{3}$.ha-1. As estimativas de Tora I e Tora II apresentaram tendeciosidade de superestimativa.

Os resultadois obtidos para o sistema de modelagem desenvolvido nesse estudo torna inviável sua aplicação em nível de escala operacional devido a baixa precisão das estimativas.

Ferraz Filho et al. (2015) citaram que existe um tradeoff entre o aumento do grau de detalhamento de um sistema de modelagem e a propagação dos erros provenientes da aplicação das diversas equações utilizadas. Essa propagação faz com que modelos mais complexos possam apresentar menor precisão e acurária na projeção do volume futuro e assim pode comprometer a tomada de decisões do manejador florestal.

Shortt \& Burkhart (1996) citam que com o aumento do período de projeção, os modelos de árvores individuais tendem a perder a precisão rapidamente. Isso se deve ao fato de que a projeção do ano seguinte é baseada no ano anterior e com isso a propagação dos erros ocorre nos anos subsequentes sendo que a indicação do autor é que projeções acima de 3 anos podem gerar erros significativos como os observados nessa pesquisa a qual teve em média 
8 projeções desde a primeira medição. Esse comportamento também foi relatado nos estudos de (QIN \& CAO, 2006).
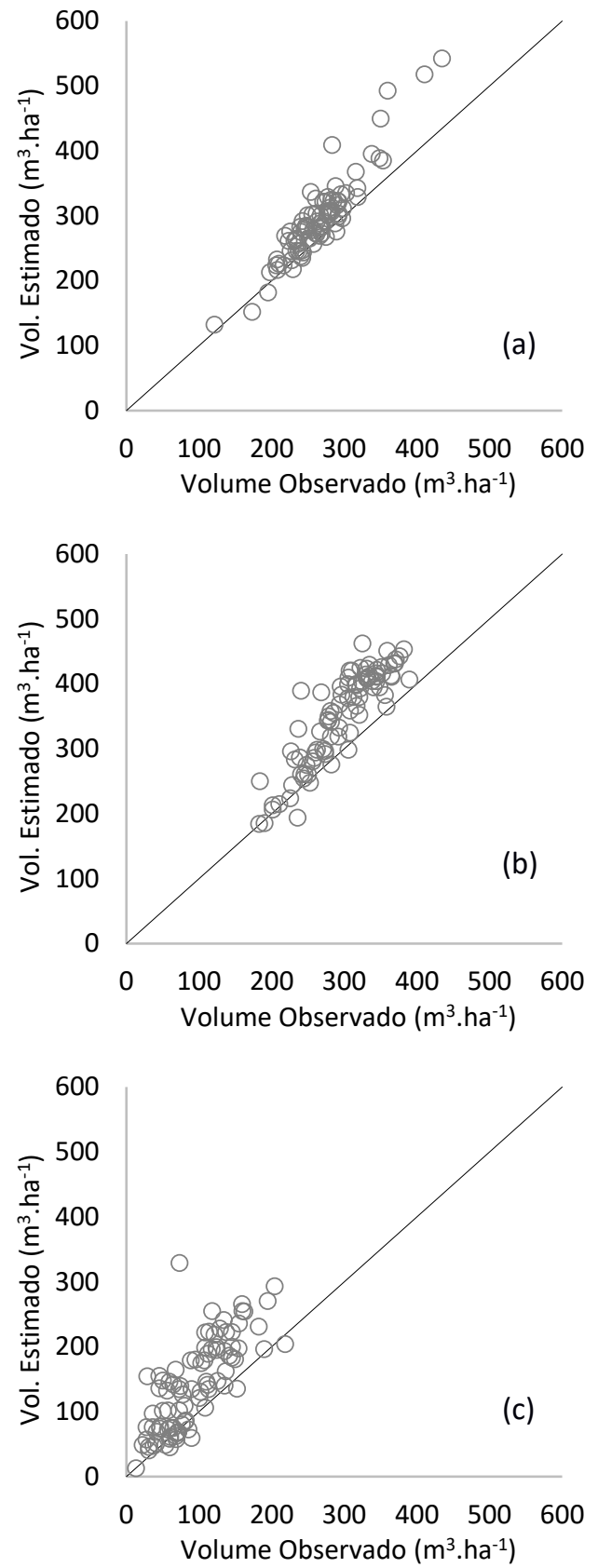

Figura 5. Volumes preditos em função dos volumes observados. (a) Volume de Celulose; (b) Volume de Tora Tipo I; (c) Volume de Tora Tipo II nos povoamentos de Pinus taeda.

A baixa precisão e acurácia apresentadas anteriormente para projeção do volume possivelmente pode ser atribuída a essa propagação dos erros, uma vez que os ajustes de cada equação do sistema apresentaram estatísticas adequadas.
Uma alternativa a mitigação da propagação de erros foi proposta por Cao (2014) com estudo de combinação entre a modelagem global e de árvores individuais a qual apresentou resultados superiores quando comparados com a modelagem tradicional com uso dos dois métodos independentes. Em estudo complementar, Cao (2019) concluiu novamente que a integração dos dois métodos proporcionou maior precisão em suas estimativas. Pesquisas complementares sobre sa combinação de classes de modelos são apresentadas por Hevia et al. (2015) e Coble et al. (2011).

\section{CONCLUSÕES}

O sistema de árvores individuais construído para prognose do volume por sortimentos não produz projeções acuradas para aplicação operacional considerando os três sortimentos avaliados, embora tenham apresentado estatísticas apropriadas nos modelos de projeção, exceto para o modelo que expressa a probabilidade da mortalidade.

Recomenda-se ajustar e testar a prognose do crescimento em períodos de no máximo três anos e desenvolver pesquisas adicionais com uso de métodos e modelos que possam expressar com maior precisão a probabilidade de mortalidade como no estudo apresentado por Cao (2017).

Torna-se oportuno desenvolver estudos que utilizem a combinação entre a modelagem de árvores individuais e a modelagem de povoamento total conforme pesquisas realizadas por CAO (2014) e CAO (2019) visando a redução da propagação de erros.

\section{REFERÊNCIAS}

ASSMANN, E. The principles of forest yield study: studies in the organic production, structure, increment and yield of forest stands. Oxford: Pergamon, 1970.

BURKHART, E.H.; TOMÉ, M. Modeling Forest Trees and Stands. New York: Springer, 2012.

CAMPOS, J.C.C.; LEITE, H.G. Mensuração florestal: perguntas e respostas. 5.ed. Viçosa: UFV, 2017.

CAO, Q.V. Linking individual-tree and whole-stand models for forest growth and yield prediction. Forest Ecosystems, v.1, p.1-8, 2014.

CAO, Q.V. Evaluation of Methods for Modeling Individual Tree Survival. Forest Science, v.63, p.356-361, 2017.

CAO, Q.V. A method to derive a tree survival model from any existing stand survival model. Canadian Journal of Forest Research, v.49, p.1598-1603, 2019. 
CHAPMAN, D.G. Statistical problems in dynamics of exploited fisheries population. 4 Berkeley Symposium on Mathematical Statistics and Probability, 1961.

COBLE, D.W. et al. An annual tree survival and diameter growth model for loblolly and slash pine plantations in East Texas. Southern Journal of Applied Forestry, v.36, p.79-84, 2011.

FERRAZ FILHO, A.C. Modeling growth and yield of loblolly pine stands under intensive management. Pesquisa Agropecuária Brasileira, v.50, n.8, p. 707-717, 2015.

FIORENTIN, L.D. et al. Generalized linear models for tree survival in loblolly pine plantations. Cerne, v.25, n.4, p.347-356, 2019.

HEVIA, A. et al. Compatibility of whole-stand and individual-tree models using composite estimators and disaggregation. Forest Science, v.348, p.46-56, 2015.

MIRANDA, R.O.V. Modelagem de árvores individuais para povoamentos não desbastados de Pinus taeda L. 2016. 168 p. (Tese de doutorado).

MIRANDA, R.O.V. et al. Modelagem da mortalidade em povamentos de Pinus taeda. Scientia Forestalis, v.45, n.115, p.435-444, 2017.

PRETZSCH, H. Forest dynamics, growth and yield. Berlin: Springer, 2009.

QIN, J.; CAO, Q.V. Using disaggregation to link individual-tree and whole-stand growth models. Canadian Journal of Forest Research, v.36, p.953-960, 2006.

RICHARDS, F.J. A flexible growth function for empirical use. Journal of Experimental Botany, v.10, n.2, p.290-301, 1959.

SCHOEPPER, W. Autimatisierung des massen, sorten and wertberechnung stenender Waaldbestande Schriftenreihe Bad. Wurtt-Forstl., 1966.

SCOLFORO, H.F. et al. Eucalyptus growth and yield system: Linking individual-tree and stand-level growth models in clonal Eucalypt plantations in Brazil. Forest Ecology and Management, v.432, p.1-16, 2019.

SHORTT, J.S; BURKHART, H.E. A comparison of loblolly pine plantation growth and yield models for inventory updating. Southern Journal of Applied Forestry, v.20, p.15-22, 1996.

STAGE, A.R. Prognosis model for stand development. USDA Forest Service: Research Paper INT-137, 1973.

TÉO, S.J. Modelagem do crescimento e produção de árvore individual independente da distância, para Pinus taeda L., na região meio oeste do estado de Santa Catarina. 2017. 272p. (Tese de doutorado). 